\section{б PATIENT \\ CHOICE}

\title{
Alleviation of carer strain during the use of the NeuroPage device by people with acquired brain injury
}

\author{
Thomas W Teasdale, ${ }^{1}$ Hazel Emslie, ${ }^{2}$ Kirsten Quirk, ${ }^{3}$ Jonathan Evans, ${ }^{3,4}$ Jessica Fish, ${ }^{2}$ \\ Barbara A Wilson ${ }^{2,3}$
}

\begin{abstract}
${ }^{1}$ Department of Psychology, University of Copenhagen, Copenhagen, Denmark; ${ }^{2}$ Medical Research Council Cognition \& Brain Sciences Unit, Cambridge, UK; ${ }^{3}$ Oliver Zangwill Centre, The Princess of Wales Hospital, Ely, UK; ${ }^{4}$ Section of Psychological Medicine, Faculty of Medicine, University of Glasgow, UK
\end{abstract}

Correspondence to:

Professor B A Wilson, Senior Scientist, Medical Research

Council Cognition and Brain

Sciences Unit, 15 Chaucer Road,

Cambridge CB2 7EF, UK:

barbara.wilson@mrc-cbu.cam.

ac.uk

Received 15 September 2008 Revised 23 January 2009 Accepted 27 January 2009

Published Online First

25 February 2009

\begin{abstract}
Background: Previous studies have demonstrated the efficacy of a paging system, NeuroPage (Cambridgeshire Primary Care Trust Corporation, Fulbourn, Cambridgeshire, UK), in compensating for memory and planning dysfunctions in people with acquired brain injury (ABl; mainly stroke or traumatic brain injury). In this study, the degree to which this efficacy is accompanied by a reduced experience of strain among carers of patients with $A B \mid$ was investigated.
\end{abstract}

Methods: Carers of 99 people with ABI completed a questionnaire concerning strain resulting from caring for the injured individual. The questionnaire was completed at the following three time points: before the use of NeuroPage, at the end of a 7-week period of use, and, for one subgroup, a further 7 weeks after withdrawal of NeuroPage.

Results: There were significant reductions in strain reported by carers following the 7-week period of NeuroPage use (Cohen's $d=0.3-0.4$ ). This finding persisted when the carer was a spouse or a parent. The reduced strain among carers continued even after withdrawal of NeuroPage.

Conclusion: The efficacy of the NeuroPage paging system for people with $A B I$ appears to result in reduced strain for their carers.

The occurrence of disability, mental or physical, in a family member who is not institutionalised and lives at home often places a strain on other members of the family who have become carers. ${ }^{1-3}$ People who have suffered an acquired brain injury (ABI; e.g. stroke or traumatic brain injury) often have difficulties with memory and planning. It is these impairments that are the greatest sources of strain to carers, ${ }^{4-7}$ who need to monitor, and perhaps assist in, the performance of everyday activities.

Electronic technology can considerably improve the performance of daily tasks in people with ABI, thus reducing their dependence on carers. ${ }^{8}$ Particular success has been reported with NeuroPage system (Cambridgeshire Primary Care Trust Corporation, Fulbourn, Cambridgeshire, UK), which involves an electronic pager worn by the person with ABI. The pager receives preprogrammed timely reminders to perform tasks-so-called 'target behaviours' - that have been specified in advance by the patient. ${ }^{910}$ Typical target behaviours include taking medicine, feeding pets, remembering appointments and remembering to take keys when leaving home.

Using the same patients who took part in the initial studies on the efficacy of NeuroPage, ${ }^{9-11}$ the present study examined whether the use of this device also results in a reduction in the strain experienced by carers.

\section{METHODS}

This study used a cross-over design that involved alternately allocating patients in blocks of 10 to one of two groups, with measurements taken at three time points over a 16-week period. Time point 1 was during a 2 -week baseline period in which both groups recorded self-identified target behaviours. During this time, a carer completed the postal strain questionnaire (the modified Caregiver Strain Index, mCSI), as described below. Group A then used the NeuroPage devices for 7 weeks and target behaviours were measured for the last 2 weeks of this period-time point 2-during which time the mCSI was completed by the carers for a second time. Group B were held on a waiting list for the same 7 weeks, during the last 2 weeks of which (also time point 2) target behaviour assessment and mCSI data were again collected. At the end of this 7-week period, NeuroPage devices were withdrawn from patients in Group A and assigned to members of Group B. For both groups, the study continued for a further 7 weeks, in which target behaviours were assessed and the mCSI completed during the final two weeks-time point 3. Further details of the study design have been reported elsewhere. ${ }^{910}$

\section{Participants}

Participants in this report were primary carers of the people with $\mathrm{ABI}$ who had been recruited to the original NeuroPage study, one carer for each person. The original study involved 143 patients with $\mathrm{ABI}$, but adequate carer data across the three time points were only available for 99 (69\%) patients, the attrition primarily due to people not having carers. Of the 99 patients with ABI, 62 had been allocated to Group A and 37 to Group B, the discrepancy arising from the block allocation of subjects and the practical constraints of completing the study. Demographic and medical characteristics of the two groups are shown in table 1 . There were no significant differences between the two groups at baseline.

\section{Instruments}

The questionnaire used to assess carer strain was a modified version of the Caregiver Strain Index, which was developed by Robinson ${ }^{12}$ and concerns 
Table 1 Demographic and medical characteristics of the patients with acquired brain injury

\begin{tabular}{lll}
\hline & Group A $(\mathbf{n}=62)$ & Group B $(\mathbf{n}=\mathbf{3 7})$ \\
\hline Gender (\%) & & \\
$\quad$ Male & 79 & 70 \\
$\quad$ Female & 21 & 30 \\
Carer (\%) & & \\
$\quad$ Spouse & 61 & 57 \\
$\quad$ Parent & 24 & 38 \\
Other & 15 & 5 \\
Type of Injury (\%) & & \\
$\quad$ Traumatic brain injury & 48 & 39 \\
Cerebrovascular accident & 25 & 25 \\
$\quad$ Other (e.g. anoxia, tumour) & 27 & 36 \\
Age at injury (mean in years (SD)) & $33(15)$ & $33(19)$ \\
Age at program entry (mean & $38(13)$ & $37(16)$ \\
in years (SD)) & & \\
\hline
\end{tabular}

"things which other people have found difficult in helping with, after someone comes home from the hospital". Our 16-item modified version was tailored towards potential stressors of particular relevance in cases of ABI. Responses were made on an 11-point scale of strain ranging from 0 (lowest) to 10 (highest). A total score was computed by averaging the $0-10$ response across the 16 items. The internal reliability of the total score was high (Cronbach's alpha $=0.91$ ).

The study complied with the Declaration of Helsinki and was approved by 11 regional ethics committees around the United Kingdom.

\section{RESULTS}

Means and standard errors of the mCSI scores for the two groups at the three time points are shown in fig 1.

The first issue examined was the test-retest reliability of the mCSI itself. During the 7 -week period between time point 1 and time point 2 , there were no systematic changes in circumstances for the 37 people in Group B. The intra-class correlation

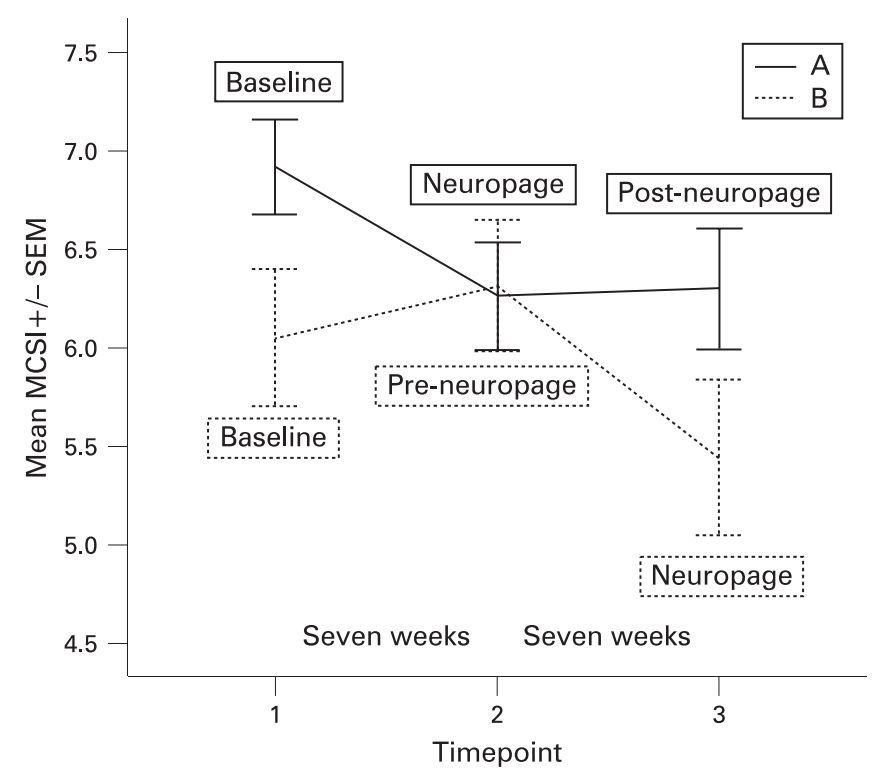

Figure 1 Mean $( \pm S E M)$ modified Caregiver Strain Index score relative to group (A or B) and time point. MCSI, modified Caregiver Strain Index. between mCSI ratings for the two time-points was 0.86 $(p<0.01)$, indicating a high level of test-retest reliability. The mean mCSI score did not change significantly for Group B between time point 1 and time point $2(t(36)=1.46, p>0.05)$. Carers in Group A reported significantly higher levels of strain at baseline than did those in Group B $(t(97)=2.11, p=0.04)$.

The overall effect of the introduction of NeuroPage was assessed by combining data from the two groups. For group A, data from time point 1 was compared with time point 2 data and for group $\mathrm{B}$, time point 2 data was compared with time point 3 data. Thus, in both cases a comparison was made between the immediate pre-Neuropage period and the end of the 7 weeks of Neuropage usage. A mixed-model analysis of variance was performed, with group as a between-subjects factor and time point as a within-subjects factor. There was no overall difference between groups $\mathrm{A}$ and $\mathrm{B}(\mathrm{F}(1,97)=3.1 ; \mathrm{p}>$ $0.05)$ and there was no significant interaction between groups and time point $(\mathrm{F}(1,97)=0.4 ; \mathrm{p}>0.05)$. On the other hand, the main effect of time point was highly significant $(F(1,97)=24.1$, $\mathrm{p}<0.001)$. The effect sizes for groups $A$ and $B$ can be estimated, using Cohen's d effect size calculation, ${ }^{13}$ as 0.33 and 0.39 respectively, which can be considered as small to medium. For both groups, over two-thirds of carers reported lower levels of strain following use of NeuroPage than before its use.

In separate matched-pairs t-tests, the reduction in strain was found to be significant for both carers who were spouses $(t(58)=3.3 ; \quad p=0.002)$ and carers who were parents $(t(28)=2.8 ; p=0.01)$. The degree of reduction in strain was similar in these two groups $(\mathrm{t}(86)=1.1 ; \mathrm{p}>0.05)$.

The final issue addressed was the extent to which the now demonstrated beneficial effect of NeuroPage was maintained after the device was withdrawn. This was examined by comparing the mCSI ratings for Group A at time point 2during NeuroPage usage-with those from time point 3-7 weeks after the device had been withdrawn. As might be expected from the means shown in figure 1, there was no significant reversal of mCSI ratings following withdrawal of NeuroPage $(t(53)=0.37 ; p>0.05)$.

\section{DISCUSSION}

Before considering these findings in detail, some limitations of the study need to be recognized. First, the information here was gathered indirectly, being obtained from relatives completing postal questionnaires. Both the internal and test-retest reliabilities are high, however, suggesting that the questionnaires were carefully and seriously completed by the carers. In addition, the motivation among carers to be part of the study was generally very high. A second potential limitation is that the relatives were not blind to the study design, being necessarily aware of when NeuroPage was in use. It is seems unlikely, however, that this knowledge would have led to a bias towards favourable responding in terms of their own strain; that is, if NeuroPage use had not in reality resulted in such a reduction. The mCSI could be criticized for only including negative items, possibly leading to response bias. A further limitation of the study is the lack of randomisation. There was a baseline difference between the two groups in the level of strain initially reported by carers, which could have been due to the (albeit non-significant) differences in the two groups' composition (i.e. Group A contained proportionately more males and more persons with traumatic brain injury). Both this difference and the discrepancy in group sizes are not critical, however, because the study design does not hinge upon group comparisons. 
Given the already reported extensive evidence for the efficacy of NeuroPage in assisting people with ABI to cope with, in particular, difficulties of memory and planning, ${ }^{10}{ }^{11}$ the present confirmation of a reduced strain for carers, both parents and spouses, is encouraging. The lack of any systematic change in reported strain in the pre-NeuroPage period for group B further supports the inference that the reduced strain experienced by carers after the introduction of the device is indeed a direct consequence of that use, rather than being some other function of the passing of time. The fact that the people with ABI in this study were on average about 5 years postinjury also makes it unlikely that the observed improvements over the 7-week period were unrelated to the use of NeuroPage. Ancillary analyses not presented here showed that the strain relief reported by carers to people with traumatic brain injury or cerebrovascular accident was greater for carers to persons who showed significant improvement in attaining targets with NeuroPage, themselves an overwhelming majority, than for the patients who did not. ${ }^{11}$

In summary, the present results suggest that not only does the use of NeuroPage improve the performance of everyday tasks among people with ABI, but that this improvement itself can result in a significant and enduring alleviation of strain in their carers.

Competing interests: None.

\section{REFERENCES}

1. Baronet AM. Factors associated with caregiver burden in mental illness: a critical review of the research literature. Clin Psychol Rev 1999;19:819-41.

2. Ferrario SR, Vitaliano P, Zotti AM, et al. Alzheimer's disease: usefulness of the Family Strain Questionnaire and the Screen for Caregiver Burden in the study of caregiving-related problems. International Journal of Geriatric Psychiatry 2003;18:1110-4.

3. Chipchase SY, Lincoln NB. Factors associated with carer strain in carers of people with multiple sclerosis. Disability and Rehabilitation 2001;23:768-76.

4. Thomsen IV. The patient with severe head injury and his family. A follow-up study of 50 patients. Scand J Rehabil Med 1974;6:180-3.

5. Livingston MG, Brooks DN, Bond MR. Three months after severe head injury: psychiatric and social impact on relatives. J Neurol Neurosurg Psychiatry 1985;48:870-5

6. Oddy M, Humphrey M, Uttley D. Stresses upon relatives of head-injured patients. British Journal of Psychiatry 1978;133:507-13.

7. Oddy M, Herbert C. Intervention with families following brain injury: evidence-based practice. Neuropsychol Rehabil 2003;13:259-73.

8. LoPresti EF, Mihailidis A, Kirsch N. Assistive technology for cognitive rehabilitation: state of the art. Neuropsychol Rehabil 2004;14:5-39.

9. Wilson BA, Emslie HC, Quirk K, et al. Reducing everyday memory and planning problems by means of a paging system: a randomised control crossover study. J Neurol Neurosurg Psychiatry 2001;70:477-82.

10. Wilson BA, Emslie $\mathrm{H}$, Quirk $\mathrm{K}$, et al. A randomized control trial to evaluate a paging system for people with traumatic brain injury. Brain Inj 2005;19:891-4.

11. Fish J, Manly T, Emslie H, et al. Compensatory strategies for acquired disorders of memory and planning: differential effects of a paging system for patients with brain injury of traumatic versus cerebrovascular aetiology. J Neurol Neurosurg Psychiatry 2008; 79:930-5

12. Robinson BC. Validation of a Caregiver Strain Index. J Gerontol 1983;38:344-8.

13. Howell DC. Statistical Methods for Psychology. New York, NY, USA: Duxbury, 2002.

\section{Access a vast information database with Toll-Free linking}

"Toll-free" linking gives you immediate access to the full text of many of the cited articles in a paper's reference list-FOR FREE. With the support of HighWire's vast journal catalogue, a huge reference library is now open to you. If HighWire hosts the journal, you can view the full text of the referenced article, completely free of charge by following the Free Full Text links in the references. 